\title{
VARIABILIDADE ESPACIAL DA RENTABILIDADE, PERDAS NA COLHEITA E PRODUTIVIDADE DO FEIJOEIRO
}

\author{
JERRY A. JOHANN ${ }^{1}$, MARIA C. A. SILVA ${ }^{2}$, MIGUEL A. URIBE-OPAZO ${ }^{3}$, \\ GUSTAVO H. DALPOSSO ${ }^{4}$
}

\begin{abstract}
RESUMO: O feijoeiro comum é cultivado em todas as regiões do País e apresenta grande importância econômica e social. É uma cultura de subsistência em pequenas propriedades, porém apresenta alguns problemas que influenciam em sua comercialização, como oscilação de preço pago ao produtor. Os métodos de colheita são diversos e, atualmente, o mais utilizado no Brasil é a colheita semimecanizada. Com o objetivo de avaliar a produtividade, rentabilidade, perdas manual e mecânica na colheita da cultivar de feijão Juriti, realizou-se o levantamento de dados georreferenciados em uma área de 10,45 ha, com o auxílio de uma grade de amostragem regular de 50 × $50 \mathrm{~m}$. Para estudar a variabilidade espacial, foram utilizadas técnicas geoestatísticas, que geraram mapas temáticos por meio da krigagem. Constatou-se que, para a safra de 2005/2006, a produtividade média encontrada na área foi $7,66 \%$ maior que a média regional informada pela SEAB do Paraná. Por ocasião da colheita, as perdas manuais foram menores $(1,47 \%)$ que as mecânicas $(7,28 \%)$ e, estas, quando somadas, representaram uma perda total de $8,75 \%$ da produtividade média da área. Pelos mapas, foi possível identificar a distribuição espacial das variáveis estudadas por toda a área estudada e verificar que, em $21,37 \%$ da área cultivada, houve prejuízo, ou seja, a rentabilidade obtida com a venda do feijão foi menor que o custo de produção, causado especialmente pela baixa produtividade da cultura. Tais informações são importantes para que o agricultor conheça a sua área de plantio, bem como avalie por meio do mapa de rentabilidade se, eventualmente, outra cultura agrícola não teria uma rentabilidade maior por área.
\end{abstract}

PALAVRAS-CHAVE: dados georreferenciados, feijão Juriti, geoestatística.

\section{SPATIAL VARIABILITY OF PROFITABILITY, HARVEST LOSSES AND PRODUCTIVITY OF BEANS}

\begin{abstract}
Common bean is grown in all regions of Brazil and has an economic and social importance. It is a subsistente crop on small farms, however, there are some problems affecting its marketing, such as fluctuation of prices paid to producers. The harvesting methods are diverse and currently the most widely used in Brazil is the semi-mechanized one. In order to evaluate productivity, profitability, manual and mechanical losses of Juriti beans cultivar, it was carried out a survey of georeferenced data in an area of 10.50 ha, with a 50 x $50 \mathrm{~m}$ grid of regular sampling. To study the spatial variability, geostatistics techniques were used to draw thematic maps by kriging techniques. It was found out that for the 2005/2006 crop, the average productivity in this area was $6.77 \%$ higher than the regional average, reported by the SEAB in Paraná. At harvest, the manual losses were lower $(1.49 \%)$ than the mechanical $(8.73 \%)$, and these, when added together, represented a total loss of $10.22 \%$ of average productivity. According to the maps, it was possible to identify the spatial distribution of variables across the studied area and register that there was a loss in $35.91 \%$ of cultivated area, which means that the beans sale profitability was less than the production cost, caused mainly by the low yield of this crop. Such information is important so that farmers know their acreage, as well as evaluate, by the profitability map, if eventually, any other crop would have a better return per area.
\end{abstract}

KEYWORDS: georeferenced data, Juriti bean, geoestatistics.

\footnotetext{
${ }^{1}$ Eng $^{\mathrm{o}}$ Agrícola, Prof. Assistente, UNIOESTE, Cascavel - PR, jerry.johann@ @otmail.com.

${ }^{2}$ Eng ${ }^{\circ}$ Agrícola, Cascavel - PR, mariaclarsi@ hotmail.com.

${ }^{3}$ Estatístico, Prof. Associado, UNIOESTE, Cascavel - PR, miguel.opazo@pq.cnpq.br.

${ }^{4}$ Matemático, Mestre em Engenharia Agrícola, UNIOESTE, Cascavel - PR, gustavodalposso@ hotmail.com.

Recebido pelo Conselho Editorial em: 6-10-2008

Aprovado pelo Conselho Editorial em: 23-5-2010
} 


\section{INTRODUÇÃO}

Atualmente, toda a produção nacional de feijão, que alcança três milhões de toneladas anuais, resume-se nas variedades carioca e preta, segundo a EMBRAPA (2005). WANDER (2005) observa que a produção brasileira de feijão tem sido insuficiente para abastecer o mercado interno, devido à redução na área plantada da ordem de 35\% nos últimos 17 anos. Mesmo com o aumento de $48 \%$ na produtividade, verificado neste período, ainda registrou-se uma diminuição de $4 \%$ na produção, não sendo suficiente para atender à demanda. É reconhecida como cultura de subsistência em pequenas propriedades, muito embora tenha havido, nos últimos 20 anos, crescente interesse de produtores com grandes áreas de terra adotando tecnologias avançadas, incluindo a colheita mecanizada.

Entretanto, o método de colheita mais utilizado atualmente é o semimecanizado, por apresentar um desempenho mais satisfatório, com respeito aos custos de colheita. $\mathrm{O}$ sistema semimecanizado proporciona baixo percentual de perdas de grãos e boa capacidade de trabalho, sendo necessária a adoção de diversos procedimentos nas fases de instalação, condução e colheita do feijoeiro. Já os sistemas mecanizados indireto e direto têm-se mostrado mais apropriados para grandes produtores (SILVA, 2000).

ZIMMERMANN et al. (1988) esclarecem que os problemas que influenciam na comercialização do produto não mudaram muito nas últimas décadas, pois a oscilação dos preços do produto pago ao agricultor continua variando de uma safra para outra. Atualmente, a comercialização, conforme observa WANDER (2005), é a mais variada possível, com predomínio de um pequeno grupo de atacadistas que concentra a distribuição da produção, gerando, muitas vezes, especulações quando ocorrem problemas na produção.

O objetivo deste trabalho foi avaliar, em uma propriedade comercial, a variabilidade espacial da produtividade, da rentabilidade e da perda manual e mecânica na colheita da cultivar de feijão Juriti. Com a estimativa do custo de produção, será possível identificar espacialmente talhões dentro da área estudada com lucro ou prejuízo para este ano/safra.

\section{MATERIAL E MÉTODOS}

O presente trabalho foi desenvolvido em uma área comercial na região de Cascavel-PR, na Comunidade Nova União, com solo do tipo Latossolo Vermelho distroférrico, localizada na latitude $24^{\circ} 56^{\prime} 14^{\prime \prime} \mathrm{S}$ e longitude 53\%19'16" W com altitude aproximada de $700 \mathrm{~m}$. O clima da região é temperado, mesotérmico e superúmido com temperatura anual média em torno de $21^{\circ} \mathrm{C}$, precipitação média avaliada em $1.940 \mathrm{~mm}$ e umidade relativa média do ar anual de $75 \%$.

A área de 10,45 ha que possui o sistema de terraceamento do tipo base larga foi cultivada no sistema de plantio convencional. A semeadura do feijão da variedade Carioca cultivar Juriti foi realizada no dia 29/09/2005, com espaçamento de 0,45 $\mathrm{m}$ entre linhas e profundidade de 0,03 m.

Foram utilizados $52,7 \mathrm{~kg} \mathrm{ha}^{-1}$ de sementes e $297 \mathrm{~kg} \mathrm{ha}^{-1}$ para adubação química. Para controle das plantas daninhas, foram realizadas duas aplicações do herbicida Basagran $\left(1,2 \mathrm{~L} \mathrm{ha}^{-1}\right)$, e para $\mathrm{o}$ controle de pragas também foram utilizadas duas aplicações, sendo uma com o inseticida Tamaron $\left(0,5 \mathrm{~L} \mathrm{ha}^{-1}\right)$ e outra com o inseticida Mertin $\left(1,2 \mathrm{~L} \mathrm{ha}^{-1}\right)$. Estas aplicações foram realizadas de forma uniforme por toda a área de estudo.

Após a semeadura do feijão, a área foi georreferenciada e medida com um aparelho de GPS da marca Trimble, modelo GeoExplorer3, com o intuito de localizar a área num sistema espacial de coordenadas. Após processamento dos dados, foram marcados os pontos para a amostragem, numa grade regular de 50 x 50 m de distância, totalizando 42 pontos amostrais na área, como apresenta a Figura 1. 


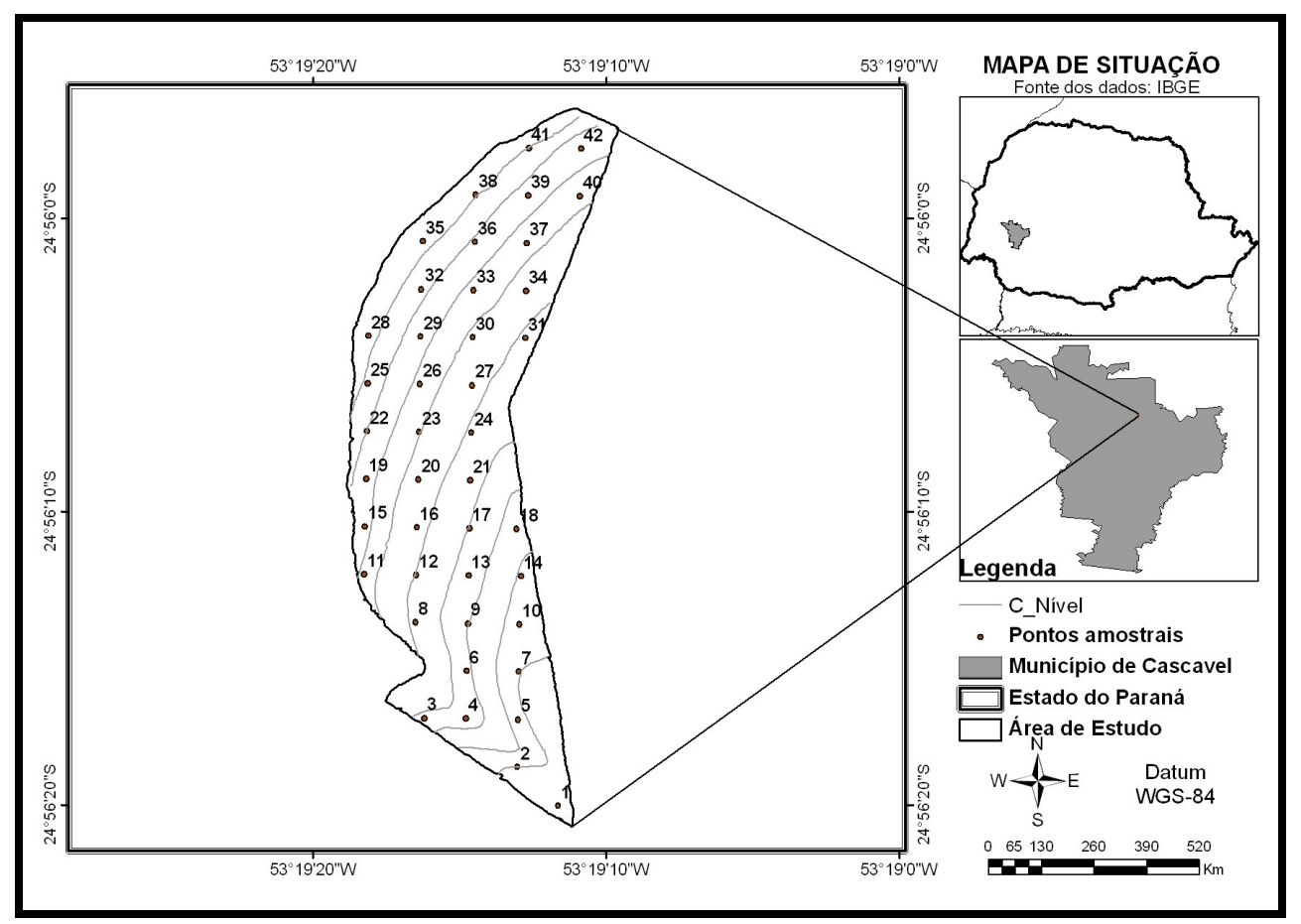

FIGURA 1. Área de pesquisa com 10,45 ha com curvas de nível e com 42 pontos de amostragem. Studied area with 10.45 ha, contour lines and 42 sampling points.

A avaliação da produtividade do feijão na área foi realizada com dois dias de antecedência da colheita da área. Assim, com o GPS, navegou-se até os 42 pontos amostrais, delimitando-se uma área de coleta de dados de 1,50 x 1,35 m (2,025 m²), da qual se realizou o arranquio do feijão. Em seguida, cada amostra foi manualmente debulhada, obtendo-se, assim, o peso de feijão para cada amostra em $\left(\mathrm{g} \mathrm{m}^{-2}\right)$ que foi convertido em $\mathrm{kg} \mathrm{ha}^{-1}$. Assim, com a produtividade de cada ponto amostral, foi possível a geração do mapa da produtividade da cultura.

Para colheita, inicialmente, foi realizado o arranquio manual de seis linhas de plantio da cultura de feijão, que em seguida foram enleiradas. Finalmente, uma recolhedora trilhadora, tracionada com trator, foi utilizada para a colheita de toda a área de estudo.

Após o arranquio do feijão e antes da passagem da máquina recolhedora trilhadora, nos pontos de coleta (com área de 1,50 x 2,70 m), todos os grãos de feijão remanescentes foram recolhidos a fim de se estimar a perda na colheita manual. Após a passagem da máquina recolhedora trilhadora, em área adjacente à anterior, porém de mesma área, foram determinadas as perdas totais na colheita, ou seja, as perdas manuais e as mecânicas. A diferença entre as perdas obtidas nas áreas de coleta, antes e após a passagem da máquina recolhedora trilhadora, foi utilizada para a determinação da perda mecânica no processo de colheita.

Após a coleta dos dados de produção por parcela e perdas na colheita manual e mecânica, os sacos que continham os grãos de feijão foram levados para pesagem $\left(\mathrm{g} \mathrm{m}^{-2}\right)$ e contagem de cada amostra. $\mathrm{Na}$ análise do custo de produção, foram considerados os gastos com sementes, fertilizantes, agrotóxicos, semeadura, pulverização, colheita e pós-colheita. Todas as atividades operacionais foram terceirizadas (semeadura, pulverização, colheita e pós-colheita).

Estas informações foram padronizadas para a unidade $\mathrm{R} \$ \mathrm{ha}^{-1}$. Para obter o lucro bruto por hectare $(L b)$, apresentado na eq.(1), considerou-se o preço do quilo de feijão em reais $(\mathrm{R} \$)$ na época em que foi comercializado. O lucro ou prejuízo $(L P)$ em cada ponto amostrado foi obtido pela diferença entre o lucro bruto $(L b)$ e o custo de produção $(C p)$ do feijão por meio da eq.(2):

$$
\begin{aligned}
& \mathrm{Lb}=\mathrm{VP} \quad \mathrm{Pd} \\
& \mathrm{LP}=\mathrm{Lb}-\mathrm{Cp}
\end{aligned}
$$


em que,

$\mathrm{Lb}$ - lucro bruto, $\mathrm{R} \$ \mathrm{ha}^{-1}$;

VP - valor do produto, $\mathrm{R} \$ \mathrm{~kg}^{-1}$;

$\mathrm{Pd}$ - produtividade, $\mathrm{kg} \mathrm{ha}^{-1}$;

LP - lucro ou prejuízo, R\$ ha ${ }^{-1}$, e

$\mathrm{Cp}$ - custo de produção, $\mathrm{R} \$ \mathrm{ha}^{-1}$.

Em seguida, realizou-se a análise estatística de todas as variáveis levantadas, considerando-se as medidas de posição e dispersão, assim como identificação da existência de pontos discrepantes (outliers), utilizando os gráficos boxplot. A produtividade encontrada na área foi comparada com os dados do Instituto Agronômico do Paraná (IAPAR) e com os dados regionais para produção da safra de 2005/2006 de feijão Juriti, obtidos pela Secretaria de Estado da Agricultura e do Abastecimento (SEAB), Departamento de Economia Rural (DERAL) e Divisão de Estatística Básica (DEB) (SEAB, 2006).

Para modelar um conjunto de dados com uma estrutura de correlação espacial, considera-se um processo estocástico Gaussiano $\{Z(s), s \in S\}$ em que $S$ é um subconjunto de $R^{2}$ que é um espaço Euclidiano bidimensional. Supõe-se que os dados $Z\left(s_{1}\right), \ldots, Z\left(s_{n}\right)$ do processo são realizações conhecidas nos locais $s_{i}, i=1, \ldots, n$,em que, $s_{i}$ é um vetor bidimensional. Supõe-se que os dados de forma geral podem ser modelados por $Z\left(s_{i}\right)=\mu_{\left(s_{i}\right)}+\mathrm{e}\left(s_{i}\right)$, sendo que $\mu_{\left(s_{i}\right)}$ é uma função determinística que descreve a componente estrutural de $Z$ em $s_{i}$ e e $\left(s_{i}\right)$ é um termo estocástico, que varia localmente e depende espacialmente de $Z\left(s_{i}\right)$. Assume-se que o termo estocástico e $\left(s_{i}\right)$ tem média zero, e a variação entre pontos no espaço é determinado pela função covariância $C\left(s_{i}, s_{j}\right)=$ $\operatorname{Cov}\left[\mathrm{e}\left(s_{i}\right), \mathrm{e}\left(s_{j}\right)\right]$ (MARDIA \& MASHALL, 1984).

A semivariância é uma função da distância $h$, que é estimada em um conjunto discreto de distâncias (lags). Em termos da covariância, a função semivariância define-se $\gamma^{(h)}=C(0)-C(h)$, sendo, $h=\left\|s_{i}-s_{j}\right\|, C(h)=C\left(s_{i}, s_{j}\right)$ e $C(0)=\sigma^{2}$ é a variância de $Z$.

A partir da estimativa de $\gamma(h)$, ajusta-se um modelo que irá depender de parâmetros desconhecidos a serem determinados e com características espaciais. Segundo ISAAKS \& SRIVASTAVA (1989), a função semivariância $\gamma(h)$ é definida na eq.(3) como:

$$
\gamma(\mathrm{h})=\frac{1}{2} \mathrm{E}[\mathrm{Z}(\mathrm{s})-\mathrm{Z}(\mathrm{s}+\mathrm{h})]^{2}
$$

em que,

$\mathrm{Z}$ (s) - valor medido em $s \in S \subset R^{2}$;

s - localização, e

h - distância que separa duas amostras.

Assim, o semivariograma experimental é um gráfico de dispersão de $\gamma(h)$ versus $h$ que permite uma análise variográfica do comportamento de $Z(s)$ (CRESSIE, 1993).

Sob as hipóteses intrínsecas, o principal estimador utilizado na construção do semivariograma experimental é o dos momentos ou conhecido como semivariograma de Matheron (CRESSIE, 1993), que é estimado pela eq.(4):

$$
\hat{\gamma}(\mathrm{h})=\frac{1}{2 \mathrm{~N}(\mathrm{~h})} \sum_{\mathrm{i}=1}^{\mathrm{N}(\mathrm{h})}[\mathrm{Z}(\mathrm{s})-\mathrm{Z}(\mathrm{s}+\mathrm{h})]^{2}
$$

em que,

$\mathrm{N}(\mathrm{h})$ - número de pares de valores medidos $\mathrm{Z}(\mathrm{s}), \mathrm{Z}(\mathrm{s}+\mathrm{h})$, separados por um vetor de distância $\mathrm{h}$;

Z(s) - variável aleatória em estudo na i-ésima posição. 
A partir da estimativa da semivariância empírica (ou experimental), ajusta-se um modelo teórico aos pontos obtidos. Escolher um modelo adequado é obter estimadores dos parâmetros efeito pepita $C_{0}$, patamar $C_{0}+C_{1}$ e alcance $a$, com métodos estatísticos de otimização, tais como: mínimos quadrados ordinários (OLS), mínimos quadrados ponderados (WLS1) (CRESSIE, 1985) e máxima verossimilhança (MV) e máxima verossimilhança restrita (MVR) (MARDIA \& MASHALL, 1984 e CHRISTENSEN et al., 1993).

Uma vez escolhido o modelo teórico de variabilidade espacial, resta saber se ele é eficiente para interpolar valores, permitindo estimativas confiáveis para a construção de mapas temáticos Para validar qual modelo ajustado é o mais adequado, utilizou-se o método de validação cruzada, que compara os valores teóricos do modelo geoestatístico escolhido e os valores empíricos obtidos na amostragem. Baseado na análise desses erros de estimação, foi selecionado o melhor modelo de semivariância (FARACO et al., 2008). Os critérios para a escolha do melhor modelo foram: valores do erro médio $(E M)$ e do erro médio reduzido $(E R)$ mais próximo de zero; o menor valor de desvio-padrão dos erros médios $\left(D P_{E M}\right)$ e do erro absoluto $(E A)$; valor de desvio-padrão dos erros reduzidos $\left(S_{E R}\right)$ mais próximos de um. As eqs. (5) a (8) representam, respectivamente, estas informações.

$$
\begin{aligned}
& \mathrm{EM}=\frac{1}{\mathrm{n}} \sum_{\mathrm{i}=1}^{\mathrm{n}}\left(\mathrm{Z}\left(\mathrm{s}_{\mathrm{i}}\right)-\hat{\mathrm{Z}}\left(\mathrm{s}_{(\mathrm{i})}\right)\right) \\
& \mathrm{ER}=\frac{1}{\mathrm{n}} \sum_{\mathrm{i}=1}^{\mathrm{n}} \frac{\mathrm{Z}\left(\mathrm{s}_{\mathrm{i}}\right)-\hat{\mathrm{Z}}\left(\mathrm{s}_{(\mathrm{i})}\right)}{\sigma\left(\hat{\mathrm{Z}}\left(\mathrm{s}_{(\mathrm{i})}\right)\right)} \\
& \mathrm{S}_{\mathrm{ER}}=\sqrt{\frac{1}{\mathrm{n}} \sum_{\mathrm{i}=1}^{\mathrm{n}} \frac{\left|\mathrm{Z}\left(\mathrm{s}_{\mathrm{i}}\right)-\hat{\mathrm{Z}}\left(\mathrm{s}_{(\mathrm{i})}\right)\right|}{\sigma\left(\hat{\mathrm{Z}}\left(\mathrm{s}_{(\mathrm{i})}\right)\right)}} \\
& \mathrm{EA}=\sum_{\mathrm{i}=1}^{\mathrm{n}}\left|\hat{\mathrm{Z}}\left(\mathrm{s}_{\mathrm{i}}\right)-\mathrm{Z}\left(\mathrm{s}_{(\mathrm{i})}\right)\right|
\end{aligned}
$$

em que,

n - número de dados;

$Z\left(s_{i}\right)$ - valor observado no ponto $s_{i}$;

$\hat{Z}\left(s_{(i)}\right)$ - valor predito por krigagem ordinária no ponto ${ }^{s_{i}}$ sem considerar a observação $Z\left(s_{i}\right)$; $\sigma\left(\hat{Z}\left(s_{(i)}\right)\right)$ - desvio-padrão da krigagem no ponto ${ }^{s_{i}}$ sem considerar a observação $Z\left(s_{i}\right)$.

A interpolação para locais não amostrados foi realizada por krigagem universal (CRESSIE, 1993). Para a análise geoestatística dos dados, foi utilizado o sistema estatístico R (IHAKA \& GENTLEMAN, 1996) e seus módulos: geoR (RIBEIRO \& DIGGLE, 2001), Splancs (ROWLINGSON \& DIGGLE, 1993) e Mass (IHAKA \& GENTLEMAN, 1996).

O grau de dependência espacial entre os elementos amostrais foi medido pelo coeficiente de efeito pepita relativo (CEPR), obtido pela Equação 9, o qual foi mencionado por CAMBARDELLA et al. (1994), que classificaram a dependência espacial como forte se $C E P R<0,25$; moderada se $0,25 \leq C E P R \leq 0,75$ e fraca se $C E P R>0,75$.

$$
\mathrm{CEPR}=\frac{\mathrm{C}_{0}}{\mathrm{C}_{0}+\mathrm{C}_{1}}
$$




\section{RESULTADOS E DISCUSSÃO}

Na Tabela 1, são apresentados os custos de produção $(C p)$ de feijão por hectare. Na safra de 2005/2006, verifica-se que da distribuição dos custos de produção, os agrotóxicos (inseticidas e herbicidas) responderam por $42,25 \%$ do custo de produção, seguido dos $24,15 \%$ de custo de colheita, dos 14,49\% de custo dos fertilizantes, dos 9,62\% do custo com sementes, dos 4,52\% com pulverização (total de 4 aplicações) e $0,70 \%$ com pós-colheita. Desta forma, os quatro primeiros itens do custo de produção representaram $90,51 \%$ dos custos totais. São estes fatores, portanto, que precisam ser avaliados pelo agricultor a fim de minimizar os custos de produção, sem prejudicar a produtividade do feijoeiro.

TABELA 1. Estimativa de custo de produção $\left(\mathrm{R} \$\right.$ ha $\left.^{-1}\right)$ da cultura do feijão na safra de 2005/2006. Production cost evaluation $\left(\mathrm{R} \$ \mathrm{ha}^{-1}\right)$ of beans in the $2005 / 2006$ harvest.

\begin{tabular}{cccc}
\hline Especificação & Custo de Produção $(C p)\left({\left.\mathrm{R} \$ \text { ha }^{-1}\right)}^{-}\right.$ & Percentual $(\%)$ & Percentagem Acumulada \\
\hline Agrotóxicos & 694,78 & 42,25 & 42,25 \\
Colheita & 397,20 & 24,15 & 66,41 \\
Fertilizantes & 238,25 & 14,49 & 80,89 \\
Sementes & 158,19 & 9,62 & 90,51 \\
Semeadura & 70,09 & 4,26 & 94,78 \\
Pulverização* & 74,40 & 4,52 & 99,30 \\
Pós-Colheita & 11,50 & 0,70 & 100,00 \\
\hline Custo total por hectare & $1.644,41$ & 100,0 &
\end{tabular}

Nota: * O custo de cada pulverização terceirizada foi de $\mathrm{R} \$ 18,60$ por hectare.

Quanto ao custo de colheita, a colheita semimecanizada utilizada tem um custo maior do que a mecanizada. Porém, como beneficio, há menor perda e quebra de grãos na colheita, agregando, assim, melhor preço ao produto final. $\mathrm{O}$ custo de $9,62 \%$ das sementes foi gerado em função da compra de sementes fiscalizadas, o que pode ser reduzido caso o agricultor utilize sementes próprias.

Na Tabela 2, é apresentada a análise exploratória da produtividade $\left(\mathrm{kg} \mathrm{ha}^{-1}\right)$, rentabilidade $\left(\mathrm{R} \$ \mathrm{ha}^{-1}\right)$, perdas manuais e mecânicas $\left(\mathrm{kg} \mathrm{ha}^{-1}\right)$ na colheita da área estudada. A produtividade do feijão foi, em média, $1.802,7 \mathrm{~kg} \mathrm{ha}^{-1}$, o que, segundo dados divulgados pelo IAPAR (2006), é uma baixa produtividade, uma vez que a produtividade média esperada desta cultivar no Paraná, na época em que foi realizado o plantio (feijão das águas), é de $2.610 \mathrm{~kg} \mathrm{ha}^{-1}$ e pode chegar a um potencial de até $3.980 \mathrm{~kg} \mathrm{ha}^{-1}$. Mas, segundo os dados da SEAB para safra de 2005/2006 de feijão das águas, a produtividade regional foi de $1.680 \mathrm{~kg} \mathrm{ha}^{-1}$ (SEAB, 2006). Assim, a área estudada apresentou uma produtividade superior à produtividade regional.

TABELA 2. Resultados estatísticos das variáveis em estudo para cultura de feijão na safra de 2005/2006. Statistical results of studied variables for beans in 2005/2006 harvest.

\begin{tabular}{ccccccccccccc}
\hline $\begin{array}{c}\text { Variáveis/ } \\
\text { Estatísticas }\end{array}$ & $\mathrm{N}^{\mathrm{o}}$ & Média & Mediana & $\mathrm{Q}_{1}$ & $\mathrm{Q}_{3}$ & $\begin{array}{c}\text { Valor } \\
\text { Mínimo }\end{array}$ & $\begin{array}{c}\text { Valor } \\
\text { Máximo }\end{array}$ & $\begin{array}{c}\text { Desvio- } \\
\text {-Padrão }\end{array}$ & $\begin{array}{c}\text { C.V. } \\
(\%)\end{array}$ & $\begin{array}{c}\text { Normalidade } \\
(\mathrm{p} \text {-valor })\end{array}$ \\
\hline $\begin{array}{c}\text { Produtividade } \\
\quad\left(\mathrm{kg} \mathrm{ha}^{-1}\right)\end{array}$ & 42 & $1.802,7$ & $1.895,4$ & $1.561,8$ & $2.068,7$ & 714,8 & $3.127,4$ & 495,6 & 27,5 & $0,221^{*}$ & $0,100^{*}$ \\
$\begin{array}{c}\text { Rentabilidade } \\
\left(\mathrm{R} \$ \text { ha }^{-1}\right)\end{array}$ & 42 & 158,3 & 251,0 & $-82,7$ & 424,3 & $-929,6$ & $1.483,0$ & 495,6 & 313,16 & $0,221^{*}$ & $0,100^{*}$ \\
$\begin{array}{c}\text { Perda manual } \\
\left(\mathrm{kg} \mathrm{ha}^{-1}\right)\end{array}$ & 42 & 26,4 & 27,5 & 15,3 & 32,9 & 0,0 & 63,4 & 16,5 & 62,3 & $0,244^{*}$ & $0,100^{*}$ \\
$\begin{array}{c}\text { Perda mecânica } \\
\left(\mathrm{kg} \mathrm{ha}^{-1}\right)\end{array}$ & 42 & 131,3 & 117,5 & 64,6 & 177,5 & 2,1 & 408,0 & 97,8 & 74,5 & 0,034 & 0,023 \\
\hline
\end{tabular}

Nota: $\mathrm{Q}_{1}$ - primeiro quartil; $\mathrm{Q}_{3}$ - terceiro quartil; C.V. - coeficiente de variação; * não se rejeita a normalidade dos dados a 5\% de significância; $A D$ - nível descritivo do teste de Anderson Darling; $S W$ - nível descritivo do teste de Shapiro-Wilk. 
Segundo Associação Brasileira de Águas Subterrâneas - ABAS (2006), a seca prejudicou quase toda a produção agrícola da safra de verão do Paraná. Segundo levantamento do Departamento de Economia Rural (DERAL) da Secretaria de Estado da Agricultura e do Abastecimento (SEAB), uma das culturas mais atingidas foi o feijão na safra das águas. Observou-se que, dos 343.531 hectares semeados com feijão, 64.253 hectares sofreram com a escassez de água, correspondendo a aproximadamente $19 \%$ da área plantada.

Para a variável rentabilidade, observa-se, pela Tabela 2, que em média o lucro por hectare foi de $\mathrm{R} \$ 158,30$, evidenciando baixa rentabilidade. Este é um dos motivos que levam muitos agricultores a desistirem do plantio do feijão, principalmente em função do alto custo de produção. A rentabilidade mediana foi $\mathrm{R} \$ 251 \mathrm{ha}^{-1}$ o que nos indica que $50 \%$ da área total teve lucro abaixo deste valor. A heterogeneidade $(\mathrm{C} . \mathrm{V} .=313,16 \%)$ encontrada para a rentabilidade evidencia que a área apresenta grande variabilidade de produtividade, já que os custos totais foram considerados os mesmos para toda a área estudada.

Ao analisar as perdas manuais de colheita, verificou-se, pela Tabela 2, que em média ela foi de $26,4 \mathrm{~kg} \mathrm{ha}^{-1}$. Considerando a produtividade média de $1.802,7 \mathrm{~kg} \mathrm{ha}^{-1}$ obtida para a área, constata-se que essas perdas manuais representaram 1,47\% da produtividade média. Este resultado foi menor que os 4,38\% encontrados por COSTA \& PASQUALETO (1999) e os 2,93\% verificados por CONTO et al. (1980), mostrando que as perdas manuais (perdas no arranquio, enleiramento manual, deiscência natural, manipulação e viragem do feijão) ocorridas por ocasião da colheita foram baixas. Já as perdas mecânicas na colheita, que foram de 131,3 $\mathrm{kg} \mathrm{ha}^{-1}$, representaram 7,28\% da produtividade média da área. Assim, em média, as perdas totais (manual + mecânica) na colheita foram de $157,7 \mathrm{~kg} \mathrm{ha}^{-1}(8,75 \%$ da produtividade média), um pouco menor do que os $8,81 \%$ encontrados por COSTA \& PASQUALETO (1999) para este sistema de colheita. Entretanto, segundo GILIOLI et al. (1991), as empresas de máquinas agrícolas têm-se esforçado em lançar novos modelos no mercado para redução das perdas na colheita, que em geral ficam entre 180 e $420 \mathrm{~kg} \mathrm{ha}^{-1}$. Com base nesta informação, verifica-se que as perdas totais médias encontradas para área foram abaixo do mínimo especificado por GILIOLI et al. (1991).

Detectou-se, de forma geral, que as variáveis em estudo apresentaram alta heterogeneidade em relação a suas médias em função de o coeficiente de variação ser maior que $20 \%$ (GOMES \& GARCIA, 2002). Além disso, à exceção da perda mecânica na colheita, as variáveis apresentaram normalidade a 5\% de significância, segundo os testes estatísticos apresentados na Tabela 2.

Observando os gráficos Boxplot da Figura 2, verifica-se que as variáveis produtividade e rentabilidade apresentaram valores discrepantes (outlier). Já a variável perda mecânica na colheita apresentou um valor discrepante unilateral superior, o que provavelmente levou à não normalidade detectada nos testes estatísticos.

$\mathrm{Na}$ Tabela 3, são apresentados os parâmetros de dependência espacial Efeito Pepita $\left(C_{0}\right)$, Contribuição $\left(C_{1}\right)$ e Alcance $(a)$ estimados pelos métodos de mínimos quadrados ordinários (OLS), mínimos quadrados ponderados (WLS1), máxima verossimilhança (MV) e máxima verossimilhança restrita (MVR). As variáveis em estudo foram modeladas com o modelo exponencial assumindo 350 m (50\% da distância máxima) como maior distância para o estudo da função semivariância (CLARK, 1979). A escolha do modelo exponencial foi realizada com base na análise do gráfico do semivariograma experimental para todas as variáveis. 

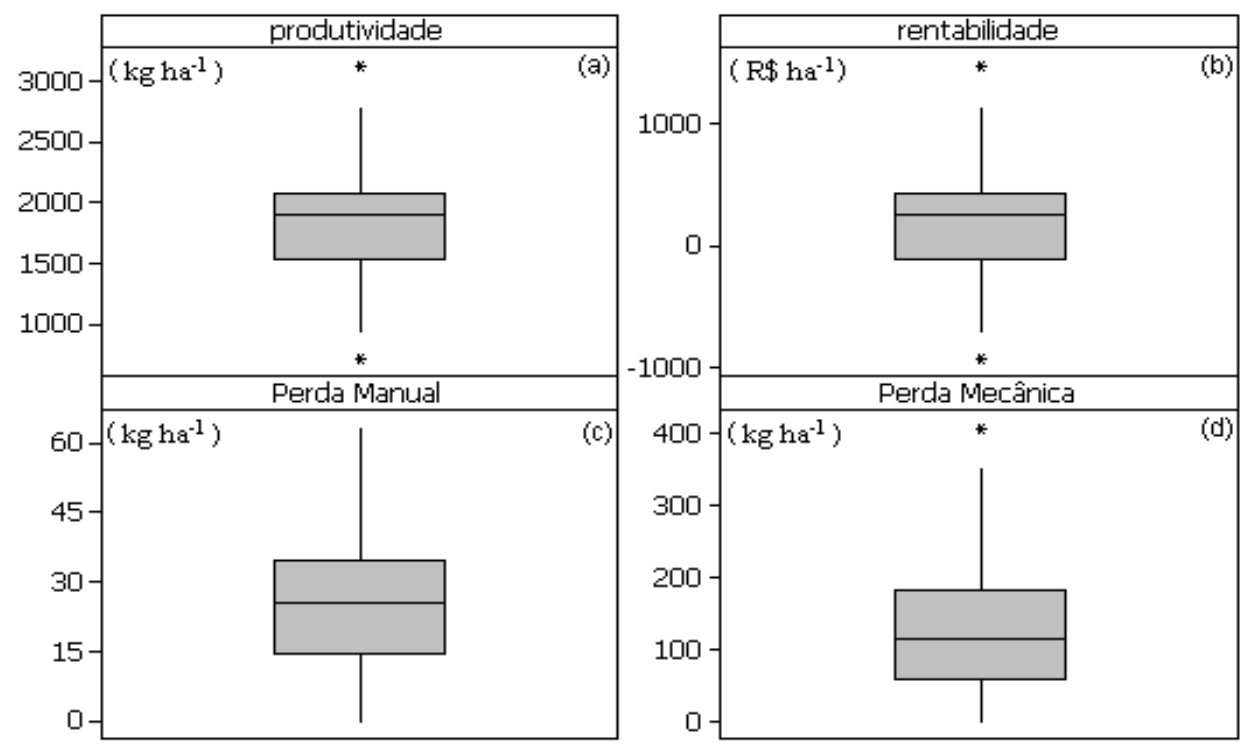

FIGURA 2. Boxplot da (a) produtividade $\left(\mathrm{kg} \mathrm{ha}^{-1}\right)$, (b) rentabilidade $\left(\mathrm{R} \$ \mathrm{ha}^{-1}\right)$, (c) perda manual na colheita $\left(\mathrm{kg} \mathrm{ha}^{-1}\right)$ e (d) perda ecânica na colheita $\left(\mathrm{kg} \mathrm{ha}^{-1}\right)$. Boxplot of (a) productivity $\left(\mathrm{kg} \mathrm{ha}^{-1}\right)$, (b) profitability $\left(\mathrm{R}_{\mathbf{S h a}}{ }^{-1}\right)$, (c) manual loss at harvest $\left(\mathrm{kg} \mathrm{ha}^{-1}\right)$ and (d) mechanical loss at harvest $\left(\mathrm{kg} \mathrm{ha}^{-1}\right)$.

TABELA 3. Parâmetros estimados do modelo exponencial ajustado pelos métodos de mínimos quadrados ordinários (OLS), mínimos quadrados ponderados (WLS1), máxima verossimilhança (MV) e máxima verossimilhança restrita (MVR) das variáveis em estudo para cultura de feijão na safra de 2005/2006. Estimated parameters of the exponential model adjusted by methods of ordinary least squares (OLS), weighted least squares (WLS1), maximum likelihood (ML) and restricted maximum likelihood (MVR) of the variables in study in common bean in 2005/2006 harvest.

\begin{tabular}{|c|c|c|c|c|c|}
\hline Variáveis & $\begin{array}{l}\text { Método de } \\
\text { Estimação }\end{array}$ & $\begin{array}{c}\text { Efeito } \\
\text { Pepita }\left(C_{0}\right)\end{array}$ & $\begin{array}{c}\text { Contribuiç̧ão } \\
\left(C_{l}\right)\end{array}$ & $\begin{array}{c}\text { Alcance }(a) \\
(\mathrm{m})\end{array}$ & $\begin{array}{c}\text { Efeito Pepita } \\
\text { Relativo }(C E P R)\end{array}$ \\
\hline \multirow{4}{*}{$\begin{array}{l}\text { Produtividade } \\
\quad\left(\mathrm{kg} \mathrm{ha}^{-1}\right)\end{array}$} & OLS & $152.805,9$ & $167.874,1$ & $1.185,1$ & 0,477 \\
\hline & WLS1 & $148.106,6$ & $157.066,9$ & 975,0 & 0,485 \\
\hline & MV & $165.951,0$ & $78.045,6$ & 251,5 & 0,680 \\
\hline & MVR & $177.273,8$ & $142.110,1$ & 893,7 & 0,555 \\
\hline \multirow{4}{*}{$\begin{array}{l}\text { Rentabilidade } \\
\left(\mathrm{R} \$ \mathrm{ha}^{-1}\right)\end{array}$} & OLS & $152.805,1$ & $167.873,0$ & $1.185,1$ & 0,477 \\
\hline & WLS1 & $148.105,5$ & $157.066,2$ & 974,7 & 0,485 \\
\hline & MV & $165.950,1$ & $78.046,5$ & 251,4 & 0,680 \\
\hline & MVR & $177.270,1$ & $142.119,2$ & 893,8 & 0,555 \\
\hline \multirow{4}{*}{$\begin{array}{l}\text { Perda Manual } \\
\qquad\left(\mathrm{kg} \mathrm{ha}^{-1}\right)\end{array}$} & OLS & 150,0 & 183,9 & 63,0 & 0,449 \\
\hline & WLS1 & 199,4 & 95,8 & 100,6 & 0,676 \\
\hline & MV & 254,6 & 15,8 & 55,2 & 0,942 \\
\hline & MVR & 250,1 & 52,3 & 781,1 & 0,827 \\
\hline \multirow{4}{*}{$\begin{array}{l}\text { Perda Mecânica } \\
\qquad\left(\mathrm{kg} \mathrm{ha}^{-1}\right)\end{array}$} & OLS & $4.110,0$ & $5.271,2$ & 384,0 & 0,438 \\
\hline & WLS1 & $3.617,0$ & $5.766,0$ & 370,5 & 0,386 \\
\hline & MV & $6.570,9$ & $3.015,7$ & $1.098,6$ & 0,685 \\
\hline & MVR & $6.459,4$ & $5.404,7$ & $1.686,0$ & 0,544 \\
\hline
\end{tabular}

Entretanto, observou-se que alguns ajustes realizados pelos diferentes métodos de estimação dos parâmetros geoestatísticos apresentaram um raio de dependência espacial maior que $700 \mathrm{~m}$ que era a distância máxima. Isto ocorreu com a produtividade e a rentabilidade quando se utilizaram os métodos de estimação de parâmetros OLS, WLS1 e MVR; com a perda manual na colheita ao utilizar o método de estimação MVR e com a perda mecânica na colheita quando foram usados os 
métodos de estimação MV e MVR, como pode ser observado na Tabela 3. Dessa forma, conforme destacam DALPOSSO et al. (2009), modelos que apresentam este comportamento devem ser descartados, pois apesar de estarem matematicamente corretos, não condizem com a realidade, pois estão considerando informações que vão além da área de estudo.

Na Tabela 4, é apresentado o estudo do melhor ajuste segundo o critério da validação cruzada para os modelos da Tabela 3. Assim, com base nos critérios de validação cruzada apresentados por FARACO et al. (2008) e as considerações sugeridas por DALPOSSO et al. (2009), os resultados indicaram como melhor ajuste o modelo exponencial com parâmetros estimados pelo método por máxima verossimilhança (MV) para produtividade $\left(\mathrm{kg} \mathrm{ha}^{-1}\right)$, rentabilidade $\left(\mathrm{R} \$ \mathrm{ha}^{-1}\right)$ e perda manual na colheita $\left(\mathrm{kg} \mathrm{ha}^{-1}\right)$ e pelo método de mínimos quadrados ordinários (OLS) para perda mecânica na colheita $\left(\mathrm{kg} \mathrm{ha}^{-1}\right)$. Os modelos e parâmetros estimados, selecionados pelo critério de validação cruzada como melhor, foram utilizados na construção de cada mapa temático pelo método da krigagem ordinária.

TABELA 4. Validação cruzada dos modelos ajustados pelos métodos de mínimos quadrados ordinários (OLS), mínimos quadrados ponderados (WLS1), máxima verossimilhança (MV) e máxima verossimilhança restrita (MVR). Cross validation of the models adjusted by the methods of ordinary least squares (OLS), weighted least squares (WLS1), maximum likelihood (ML) and restricted maximum likelihood (MVR).

\begin{tabular}{|c|c|c|c|c|c|c|}
\hline Variável & $\begin{array}{l}\text { Método de } \\
\text { Estimação }\end{array}$ & $E M$ & $E R$ & $D P_{E M}$ & $S_{E R}$ & $E A$ \\
\hline \multirow{4}{*}{$\begin{array}{l}\text { Produtividade } \\
\qquad\left(\mathrm{kg} \mathrm{ha}^{-1}\right)\end{array}$} & OLS & $-5,404000$ & $-0,005848$ & 490,881534 & 1,100068 & $15.210,450$ \\
\hline & WLS1 & $-5,406000$ & $-0,005862$ & 491,246809 & 1,105749 & $15.219,900$ \\
\hline & MV & $\underline{-2,535000}$ & $\underline{-0,002628}$ & $\underline{487,760475}$ & $\underline{\mathbf{1}, 024145}$ & $\underline{15.268,220}$ \\
\hline & MVR & $-4,808000$ & $-0,004890$ & 489,434821 & 1,024673 & $15.205,780$ \\
\hline \multirow{4}{*}{$\begin{array}{l}\text { Rentabilidade } \\
\quad\left(\mathrm{R} \$ \mathrm{ha}^{-1}\right)\end{array}$} & OLS & $-5,404000$ & $-0,005848$ & 490,881534 & 1,100068 & $15.210,450$ \\
\hline & WLS1 & $-5,406000$ & $-0,005862$ & 491,246809 & 1,105749 & $15.219,900$ \\
\hline & MV & $-2,535000$ & $-0,002628$ & 487,760475 & 1,024145 & $15.268,220$ \\
\hline & MVR & $-4,808000$ & $-0,004890$ & 4899,434821 & 1,024673 & $15.205,780$ \\
\hline \multirow{4}{*}{$\begin{array}{l}\text { Perda Manual } \\
\quad\left(\mathrm{kg} \mathrm{ha}^{-1}\right)\end{array}$} & OLS & $-0,006835$ & $-0,000199$ & 17,0167537 & 0,994074 & 560,2108 \\
\hline & WLS1 & $-0,013510$ & $-0,000388$ & 17,0062460 & 0,989961 & 561,8970 \\
\hline & MV & $-0,000447$ & $-0,000013$ & $\underline{17,0429700}$ & $\underline{1,204196}$ & $\underline{559,5699}$ \\
\hline & MVR & $-0,046100$ & $-0,001360$ & 16,8045320 & $\overline{1,010286}$ & $\overline{560,1943}$ \\
\hline \multirow{4}{*}{$\begin{array}{l}\text { Perda Mecânica } \\
\quad\left(\mathrm{kg} \mathrm{ha}^{-1}\right)\end{array}$} & OLS & $\underline{-0,387700}$ & $-0,002422$ & 94,9691810 & 1,163191 & $2.900,281$ \\
\hline & WLS1 & $-0,442700$ & $-0,002833$ & $\overline{96,3448990}$ & $\overline{1,207369}$ & $\overline{2.935,878}$ \\
\hline & MV & $-0,128900$ & $-0,000757$ & 88,4678490 & 1,014995 & $2.709,579$ \\
\hline & MVR & $-0,086560$ & $-0,000534$ & 88,4927190 & 1,013566 & $2.708,498$ \\
\hline
\end{tabular}

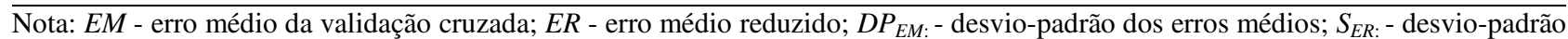
dos erros reduzidos; $E A$ - erro absoluto.

O grau de dependência espacial entre elementos amostrais das variáveis em estudo, medidos pelos CEPR dos modelos selecionados, apresentados na Tabela 3, foi classificado como dependência espacial moderada para a produtividade, rentabilidade e perda mecânica na colheita $(0,25 \leq C E P R \leq 0,75)$ e fraca para a perda manual na colheita $(C E P R>0,75)$. Com base nisto, pode-se dizer que a perda manual na colheita teve média autocorrelação espacial entre os elementos amostrais, e as demais variáveis apresentaram baixa autocorrelação espacial.

$\mathrm{Na}$ Figura 3, apresenta-se o mapa da produtividade do feijão $\left(\mathrm{kg} \mathrm{ha}^{-1}\right)$ que evidencia a variabilidade espacial encontrada na área. $\mathrm{Na}$ Tabela 5, são apresentadas as proporções de áreas para cada intervalo da produtividade. Observa-se que $41,44 \%$ da área total tiveram produtividade entre 1840 e $2.110 \mathrm{~kg} \mathrm{ha}^{-1}$, ou ainda, que $81,51 \%$ da área cultivada apresentou produtividade entre 1.570 e $2.380 \mathrm{~kg} \mathrm{ha}^{-1}$. A produtividade média obtida pelo mapa interpolado $\left(1.863,18 \mathrm{~kg} \mathrm{ha}^{-1}\right)$ foi 
superior à produtividade média da região $\left(1.680 \mathrm{~kg} \mathrm{ha}^{-1}\right)$ informada pela SEAB (2006), e inferior à produtividade média estadual $\left(2.610 \mathrm{~kg} \mathrm{ha}^{-1}\right)$, informada por IAPAR (2006). Considerando toda a área cultivada, foi possível constatar que 76,34\% dela apresentaram produtividade superior ao valor médio regional. Esta menor produtividade em relação à média estadual ocorreu, conforme já destacado (ABAS, 2006), em função da estiagem durante o ciclo fenológico da cultura.

Ao analisar a distribuição espacial da rentabilidade do feijão $\left(\mathrm{R} \$ \mathrm{ha}^{-1}\right)$ no mapa da Figura 4, verifica-se que algumas regiões da área mostraram rentabilidades negativas, ou seja, prejuízo. Dentre elas, uma localiza-se ao centro do talhão e outra na parte superior do talhão (áreas mais claras e hachuradas do mapa).

Segundo as informações apresentadas na Tabela 6, estas duas áreas totalizaram 2,23 ha, o que representa $21,37 \%$ da área total cultivada. Por consequência, no restante da área $(78,63 \%)$, obtevese lucro na produção da cultura. Evidentemente, as áreas que apresentaram menor produtividade foram as que apresentaram prejuízo $\left(\mathrm{R} \$ \mathrm{ha}^{-1}\right)$, e as que tiveram maior produtividade apresentaram, por consequência, maiores lucros. O prejuízo máximo obtido foi de $\mathrm{R} \$$ - 614,00 por hectare, e o lucro máximo foi de $\mathrm{R} \$ 1.012,00$ por hectare, porém a rentabilidade média obtida pelo mapa interpolado foi de $\mathrm{R} \$ 220,89 \mathrm{ha}^{-1}$.

É importante destacar a semelhança na variabilidade espacial dos mapas de produtividade e rentabilidade apresentados nas Figuras 3 e 4 . A explicação para isto é que a rentabilidade é uma função linear da produtividade (eq.(2)). Dessa forma, os mapas obtidos por krigagem foram semelhantes.

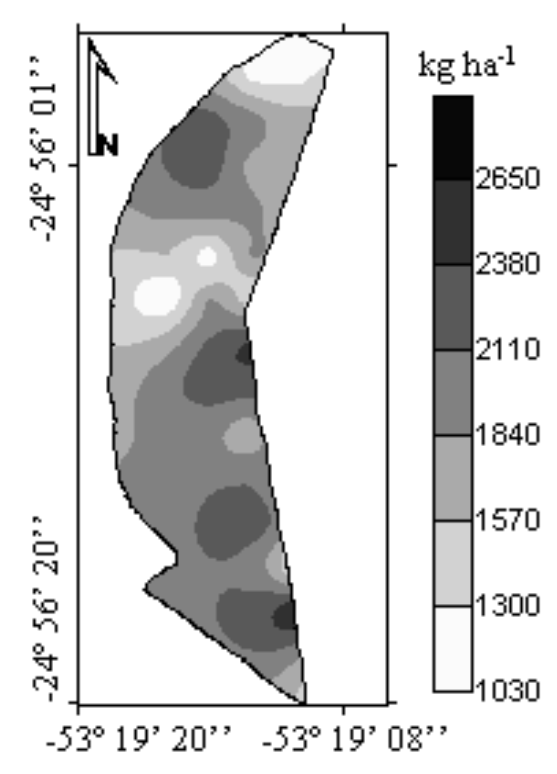

FIGURA 3. Mapa da distribuição espacial da produtividade do feijão $\left(\mathrm{kg} \mathrm{ha}^{-1}\right)$. Spatial distribution map of beans productivity $\left(\mathrm{kg} \mathrm{ha}^{-1}\right)$. 
TABELA 5. Distribuição de frequência da produtividade do feijão $\left(\mathrm{kg} \mathrm{ha}^{-1}\right)$ em relação à área total. Frequency distribution of beans productivity $\left(\mathrm{kg} \mathrm{ha}^{-1}\right)$ according to the total area.

\begin{tabular}{ccc}
\hline Intervalo de Produtividade $(P d)$ & Área $($ ha $)$ & Percentagem da Área Total \\
\hline $1030<P d \leq 1300$ & 0,612 & 5,86 \\
$1300<P d \leq 1570$ & 1,232 & 11,80 \\
$1570<P d \leq 1840$ & 2,194 & 20,99 \\
$1840<P d \leq 2110$ & 4,330 & 41,44 \\
$2110<P d \leq 2380$ & 1,994 & 19,08 \\
$2380<P d \leq 2650$ & 0,087 & 0,83 \\
$2650<P d \leq 2656$ & 0,001 & 0,00 \\
\hline Total & 10,45 & 100 \\
\hline
\end{tabular}

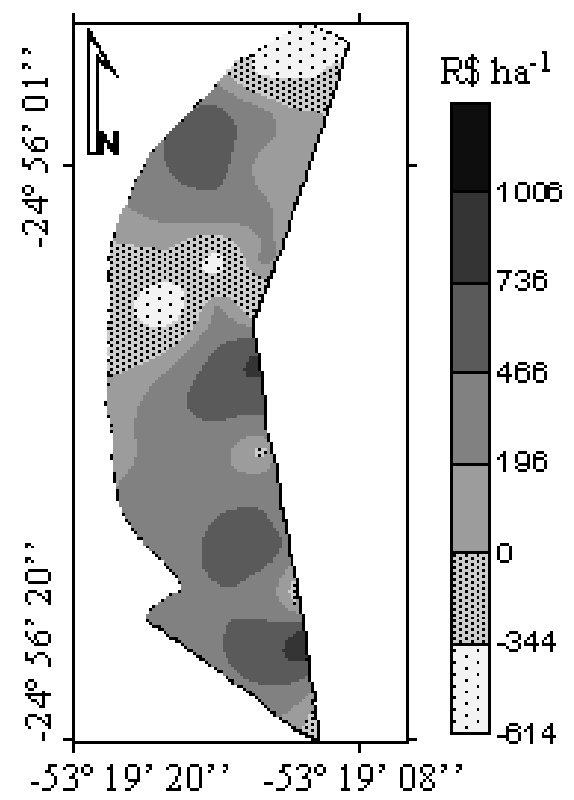

FIGURA 4. Mapa da distribuição espacial da rentabilidade do feijão ( $\left.\mathrm{R} \$ \mathrm{ha}^{-1}\right)$. Spatial distribution map of beans profitability $\left(\mathrm{R} \$ \mathrm{ha}^{-1}\right)$.

TABELA 6. Distribuição de frequência da rentabilidade do feijão $\left(\mathrm{R} \$ \mathrm{ha}^{-1}\right)$ em relação à área total. Frequency distribution of beans profitability $\left(\mathrm{R} \$ \mathrm{ha}^{-1}\right)$ according to the total area.

\begin{tabular}{ccc}
\hline Intervalo de Rentabilidade $(R)$ & Área (ha) & Percentagem da Área Total \\
\hline$-614<R \leq-344$ & 0,613 & 5,87 \\
$-344<R \leq 0$ & 1,620 & 15,50 \\
$0<R \leq 196$ & 1,811 & 17,33 \\
$196<R \leq 466$ & 4,331 & 41,46 \\
$466<R \leq 736$ & 1,987 & 19,02 \\
$736<R \leq 1006$ & 0,087 & 0,82 \\
$1006<R \leq 1012$ & 0,001 & 0,00 \\
\hline Total & 10,45 & 100 \\
\hline
\end{tabular}

Na Figura 5 e Tabela 7, são apresentados, respectivamente, o mapa e a distribuição de frequência da variável perda manual na colheita $\left(\mathrm{kg} \mathrm{ha}^{-1}\right)$. Pelo mapa, foi possível verificar a variabilidade espacial da perda manual na colheita na área em estudo. Em média, pelo mapa interpolado, ela foi de $26,69 \mathrm{~kg} \mathrm{ha}^{-1}$. Pela Tabela 7 , constata-se que, em 36,14\% da área, a perda manual na colheita variou de 20,82 a $30,82 \mathrm{~kg} \mathrm{ha}^{-1}$ e, em $87,67 \%$ de toda área, ela variou entre 10,82 e $40,82 \mathrm{~kg} \mathrm{ha}^{-1}$. 


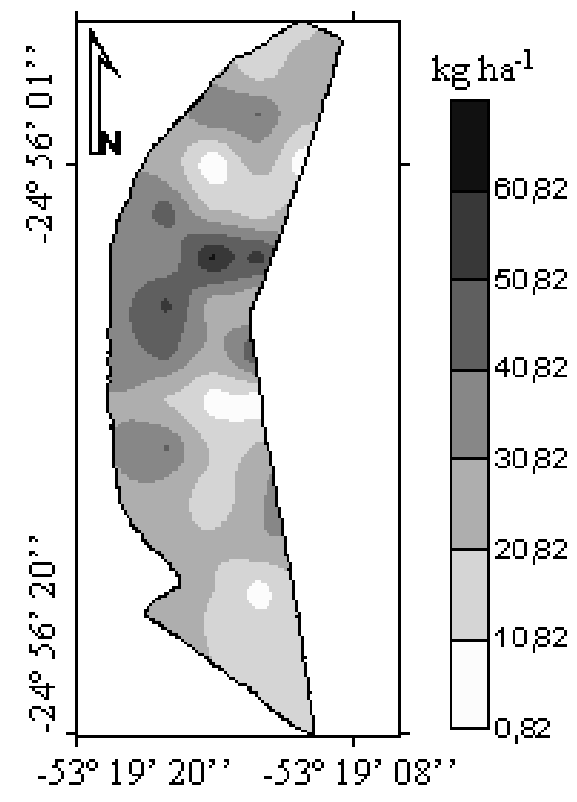

FIGURA 5. Mapa da distribuição espacial da perda manual na colheita $\left(\mathrm{kg} \mathrm{ha}^{-1}\right)$. Spatial distribution map of manual loss at harvest $\left(\mathrm{kg} \mathrm{ha}^{-1}\right)$.

TABELA 7. Distribuição de frequência da perda manual na colheita $\left(\mathrm{kg} \mathrm{ha}^{-1}\right)$ em relação à área total. Frequency distribution of manual loss at harvest $\left(\mathrm{kg} \mathrm{ha}^{-1}\right)$ according to the total area.

\begin{tabular}{ccc}
\hline Intervalo de perdas manuais $(P m)$ & Área $($ ha) & Porcentagem da área total \\
\hline $0,82<P m \leq 10,82$ & 0,328 & 3,14 \\
$10,82<P m \leq 20,82$ & 2,927 & 28,02 \\
$20,82<P m \leq 30,82$ & 3,776 & 36,14 \\
$30,82<P m \leq 40,82$ & 2,457 & 23,51 \\
$40,82<P m \leq 50,82$ & 0,844 & 8,08 \\
$50,82<P m \leq 60,82$ & 0,114 & 1,08 \\
$60,82<P m \leq 63,00$ & 0,004 & 0,03 \\
\hline Total & 10,45 & 100 \\
\hline
\end{tabular}

Já as informações das perdas mecânicas obtidas na colheita semimecanizada do feijão são apresentadas na Tabela 8 e Figura 6. Pela Figura 6, visualiza-se que, na parte sul do mapa, estão as menores perdas mecânicas e, na parte norte, as maiores perdas mecânicas. Este comportamento pode ser explicado pela declividade do terreno, já que a parte sul apresenta menor declividade, e à medida que se desloca para a parte norte da área, há um aumento na declividade do terreno, aumentando, assim, as perdas mecânicas no processo de colheita semimecanizada.

Pela Tabela 8, pode-se verificar que ela variou de 6 a $401 \mathrm{~kg} \mathrm{ha}^{-1}$. Entretanto, em 73,42\% da área, ela esteve entre 66 e $186 \mathrm{~kg} \mathrm{ha}^{-1}$, e em 97,47\% da área, as perdas mecânicas na colheita foram menores que $246 \mathrm{~kg} \mathrm{ha}^{-1}$.

Na Tabela 9, apresenta-se uma comparação dos valores médios obtidos dos dados amostrais (42 elementos amostrais) e dos valores médios obtidos das análises espaciais, utilizando os mapas interpolados por krigagem. 


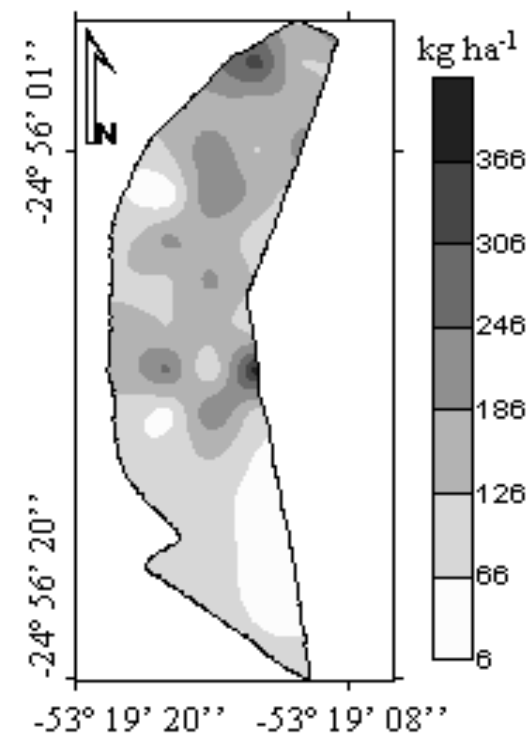

FIGURA 6. Mapa da distribuição espacial da perda mecânica na colheita $\left(\mathrm{kg} \mathrm{ha}^{-1}\right)$. Spatial distribution map of mechanical loss at harvest $\left(\mathrm{kg} \mathrm{ha}^{-1}\right)$.

TABELA 8. Distribuição de frequência da perda mecânica na colheita $\left(\mathrm{kg} \mathrm{ha}^{-1}\right)$ em relação à área total. Frequency distribution of mechanical loss at harvest $\left(\mathrm{kg} \mathrm{ha}^{-1}\right)$ according to the total area.

\begin{tabular}{ccc}
\hline Intervalo da perda mecânica $(P M)$ & Área (ha) & Porcentagem da área total \\
\hline $6<P M \leq 66$ & 1,347 & 12,89 \\
$66<P M \leq 126$ & 3,572 & 34,19 \\
$126<P M \leq 186$ & 4,099 & 39,23 \\
$186<P M \leq 246$ & 1,166 & 11,16 \\
$246<P M \leq 306$ & 0,212 & 2,02 \\
$306<P M \leq 366$ & 0,049 & 0,47 \\
$366<P M \leq 401$ & 0,005 & 0,04 \\
\hline Total & 10,449 & 100 \\
\hline
\end{tabular}

Quando comparados com os dados obtidos dos pontos amostrais, verificou-se que as variáveis produtividade, rentabilidade e perdas manuais foram, em média, superestimadas em 3,01\%;39,54\% e $1,02 \%$, respectivamente. Já as perdas mecânicas durante a colheita foram subestimadas em $0,92 \%$. A diferença observada entre as médias da rentabilidade pode ser explicada pelo alto coeficiente de variação encontrado $(\mathrm{CV}=313,16 \%)$, ou seja, devido à grande variabilidade encontrada para os dados amostrados.

TABELA 9. Valores médios dos dados amostrados e previstos utilizando os mapas interpolados. Average values of sampled and foreseen data based on interpolated maps.

\begin{tabular}{ccc}
\hline Variáveis & Média Amostral & $\begin{array}{c}\text { Valor Médio Previsto } \\
\text { Utilizando os Mapas Interpolados }\end{array}$ \\
\hline Produtividade $\left(\mathrm{kg} \mathrm{ha}^{-1}\right)$ & $1.808,70$ & $1.863,18$ \\
Rentabilidade $\left(\mathrm{R} \mathrm{ha}^{-1}\right)$ & 158,30 & 220,89 \\
Perda Manual $\left(\mathrm{kg} \mathrm{ha}^{-1}\right)$ & 26,42 & 26,69 \\
Perda Mecânica $\left(\mathrm{kg} \mathrm{ha}^{-1}\right)$ & 131,30 & 130,09 \\
\hline
\end{tabular}




\section{CONCLUSÕES}

Pelos mapas obtidos, foi possível verificar a distribuição espacial de cada variável no campo, observando-se regiões, com maior ou menor produtividade, lucro ou prejuízo e perdas manuais e mecânicas na colheita. A produtividade média da área estudada foi menor em relação à prevista pelo IAPAR, mas em relação às médias regionais, para a safra de 2005/2006 e para esta época de plantio, a cultura teve produtividade média 7,66\% acima da média regional. As perdas totais encontradas por ocasião da colheita do feijão foram baixas quando comparadas aos demais trabalhos encontrados na literatura. A rentabilidade média de 158,30 $\mathrm{R} \$ \mathrm{ha}^{-1}$ foi baixa, cerca de um terço de um salário mínimo por hectare. Além disto, foi possível verificar que, com os custos de produção e a produtividade encontrada, houve prejuízo em $21,37 \%$ da área cultivada.

A partir dos mapas de variabilidade espacial de produtividade e rentabilidade, será possível investigar os motivos das baixas produtividades em algumas regiões da área estudada, sugerindo eventualmente um estudo das propriedades físicas do solo, bem como da fertilidade do solo, no intuito de encontrar e corrigir o problema que levou às baixas produtividades nestas regiões.

\section{AGRADECIMENTOS}

Ao Conselho Nacional de Desenvolvimento Científico e Tecnológico - CNPq, CAPES e Fundação Araucária, pelo apoio financeiro.

\section{REFERÊNCIAS}

ABAS. ASSOCIAÇÃO BRASILEIRA DE ÁGUAS SUBTERRÂNEAS. Lavouras do Paraná ainda sofrem com falta de chuva. GAZETA MERCANTIL SP, AGRONEGÓCIO, 19 janeiro de 2006. Disponível em <http://www.abas.org.br/index.php>. Acesso: 06 dez. 2006.

CAMBARDELLA, C. A.; MOORMAN, T. B.; NOVAK, J. M.; PARKIN, T. B.; KARLEN, D. L.; TURCO, R. F.; KONOPKA, A. E. Field-scale variability of soil properties in central Iowa soils. Soil Science Society America Journal, Madison, v.58, n.5, p.1.501-1.511, 1994.

CHRISTENSEN, R.; JOHNSON, W.; PEARSON, L.M. Covariance function diagnostics for spatial linear models. Mathematical Geology, New York, v.25, n.2, p.145-160, 1993.

CLARK, I. Practical geostatistics. London: Applied Science Publishers, 1979. 130 p.

CONTO, A.J.; VIEIRA, E.H.N.; OLIVEIRA, E.T.; PORTES, E.; CASTRO, T.A. Aspectos técnicos e econômicos da colheita mecânica e manual do feijão (Phaseolus vulgaris $L$.). CNPAF-Embrapa. 18 p. 1980 (Circular Técnica, 2)

COSTA, L.R.M.; PASQUALETO, A. Comparação de sistemas de colheita mecanizada e semimecanizada na perda, dano mecânico e impureza de grãos na cultura do feijoeiro (Phaseolus vulgaris L.). Pesquisa Agropecuária Tropical, Goiânia, v.29, n.1, p.35-38, 1999.

CRESSIE, N.A. Statistics for spacial data. New York: John Wiley, 1993. 900 p.

CRESSIE, N. Fitting variogram models by weighted least squares. Mathematical Geology, New York, v. 17, n.4, p.563-586, 1985.

DALPOSSO, G.H. ; URIBE-OPAZO, M.A.; BORSSOI, J.A. ; JOHANN, J.A.; MERCANTE, E. Previsão da produção de Trigo utilizando métodos geoestatísticos. In: AVANCES EN INGENIERÍA RURAL 2007-2009. Rosario: Editorial de la Universidad Nacional de Rosario, 2009. v.1, p.78-86.

EMBRAPA AGÊNCIA DE INFORMAÇÃO DO FEIJÃO. 2005. Disponível em: $<$ http://www.cnptia.embrapa.br>. Acesso em: 26 maio 2006.

FARACO, M.A.; URIBE-OPAZO, M.A.; SILVA, E.A.A. da; JOHANN, J.A. ; BORSSOI, J.A. Seleção de modelos de variabilidade espacial para elaboração de mapas temáticos de atributos 
físicos do solo e produtividade da soja. Revista Brasileira de Ciência do Solo, Viçosa - MG, v.32, n.2, p.463-476, 2008.

GILIOLI, J.L.; TRECENTI, R.; TERASAWA, F.; WILLEMANN, W. Feijão, colheita mecanizada, novas cultivares FT-Rio Preto e FT-Zebrinha. Brasília: FT-Pesquisa e Sementes, 1991. 16 p.

GOMES, F.P.; GARCIA, C.H. Estatística aplicada a experimentos agronômicos e florestais. Piracicaba: FEALQ, 2002. 309 p.

IAPAR. Instituto Agronômico do Paraná. Cultivares de feijão. Londrina, 2006. Disponível em: <Www.pr.gv.br/iapar>. Acesso em: maio 2006.

IHAKA, R.; GENTLEMAN, R. A language for data analysis and graphics. Journal of Computational and Graphical Statistics, Salt Like City, v.5, n.3, p.229-314, 1986. Software.

ISAAKS, E.H.; SRIVASTAVA, R.M. An introduction to applied geostatistics. New York: Oxford University Press, 1989. $561 \mathrm{p}$.

MARDIA, K.V.; MARSHALL, R.J. Maximum likelihood models for residual covariance in special regression. Biometrika, Oxford, v.71, n.1, p.135-146, 1984.

RIBEIRO JR., P.J.; DIGGLE, P.J. GeoR: a package for geostatistical analysis. R-NEWS, v.1, n.2, p.1.609-3.631, 2001.

ROWLINGSON, B.; DIGGLE, P.J. Splancs: spatial point pattern analysis code in S-Plus. Computers and Geosciences, Canadá, v.19, n.5, p.627-655, 1993.

SEAB. SECRETARIA DA AGRICULTURA E DO ABASTECIMENTO DO PARANÁ. Estimativa de custo de produção do feijão das águas - Safra 2005/2006. 2006. Disponível em: <www.pr.gov.br/seab/deral /cp_feij\%E3o.xls>. Acesso em: 27 jun. 2006.

SILVA, J.G. da; AIDAR, H.; BEDISCHI, L.C.; MARDEGAM, F.J.; FONSECA, J.R. Mecanização da colheita do feijoeiro uso de recolhedoras trilhadoras. Santo Antônio de Goiãs: Embrapa Arroz e Feijão, 2000. Disponível em: <http://www.agencia.cnptia.embrapa.br/ Agencia4/AG01/Abertura.html>. Acesso em: 15 dez. 2005.

WANDER, A.E. Cultivo do feijão irrigado na região noroeste de Minas Gerais. Santo Antônio de Goiás: Embrapa Arroz e Feijão, 2005. Disponível em: <http://sistemasdeproducao. cnptia.embrapa.br/FontesHTML/Feijao/FeijaoIrrigadoNoroesteMG>. Acesso em: jun. 2006.

ZIMMERMANN, M.J.de O.; ROCHA, M.; YAMADA, T. Cultivo do feijoeiro: fatores que afetam a produtividade. Piracicaba: Potafos, 1988. 589 p. 